Please send trade news information and illustrations to Arveen Bajaj at the BDJ, 64 Wimpole Street, London W1G 8YS.

Trade news is supplied as a service to the reader and does not imply endorsement by the BDJ. Normal and prudent research should be exercised before purchase of use of any product mentioned.

\section{CD special offer} personal CD player with every order of two $750 \mathrm{~g}$ tubs of Alkazyme. Decontamination with Alkazyme Enzymatic rapidly dissolves blood, pus, saliva etc, leaving instruments brilliantly clean.

Alkazyme dissolves in ordinary tap water for use in any soaking bath, tray or ultrasonic bath. Its dual action cleans and disinfects all immersable instruments in minutes, without manual scrubbing.

Alkazyme is active against pathogenic gram+ and gram- bacteria, and reduces the risks of bactericidal contamination within the surgery environment, disinfecting the wash water in soaking baths/sinks etc created by the decontamination process.

Reader response number 50

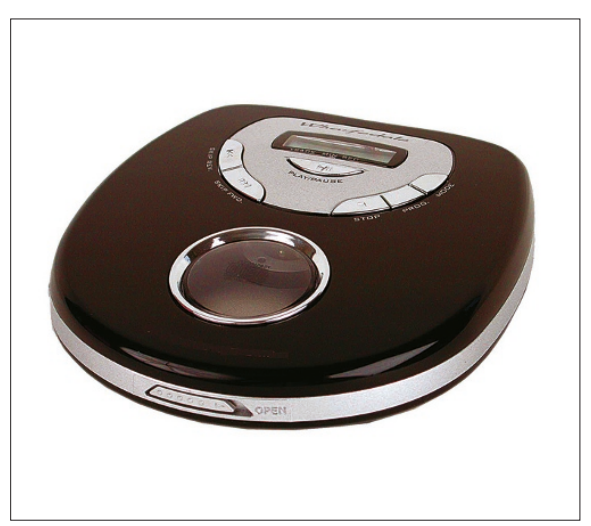

Alkapharm is running a special promotion throughout July \& August offering a free

\section{High tech camera launched}

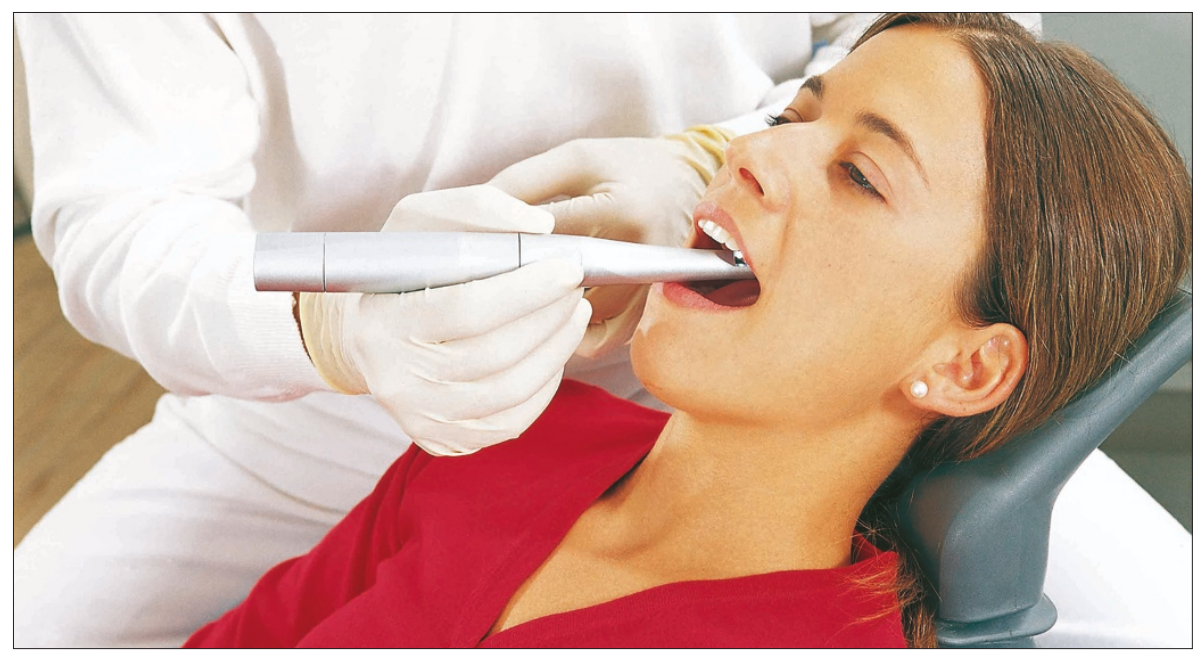

Minerva Dental has launched the new Durr VistaCam CL, the world's first mobile intraoral camera. Easy to integrate into the chair, its plug and play facility means that one camera can be used for several surgeries.

The camera is the world's first wireless camera with up to 45 minutes operating time. It features Durr IFC optics and enables dentists to go from room to room and patient to patient, offering increased freedom in diagnostics and communication.

It combines six very sensitive LEDs with a light-collecting lens, a superb depth of field and an option to use mirrored or correct reading settings. Manufactured from composite plastic it has no galvanic effects, does not feel cold and does not transmit impacts to the optics. Its lightweight and ergonomically designed handpiece also has a shifted centre of gravity to allow for handling without fatigue. It is narrow for easy access to the rear of the mouth, and all the settings such as left to right reversed display, channel selection and on/off can be activated directly at the handpiece. Finally the rechargeable battery can be swapped for a connecting cable when required, for example when recharging. This enables its continuous use throughout the day.

Reader response number 51

\section{New improved putty in your hands}

Elite H-D putty and wash has been upgraded to Elite HD+. The upgraded version features cartridges in a new $50 \mathrm{ml}$ standard with smaller mixing tips to minimise wastage. The soft consistency of the putty ensures less of the light body is disturbed from the preparation, resulting in a detailed impression.

Elite also has a snap set with a total time in the mouth of $2^{1 / 2}$ minutes. Its rigid final set minimises the possibility of distortion and offers excellent tear resistance and adhesion with the

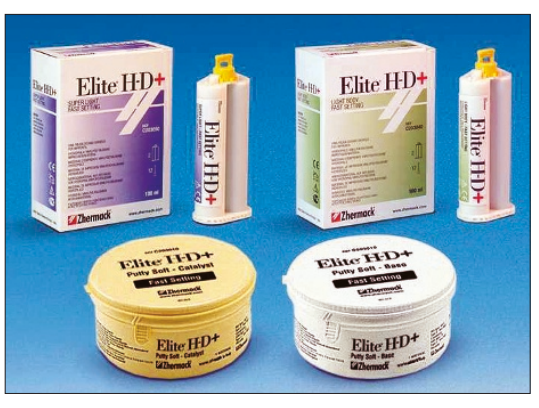
wash. Also available are new Monophase and Tray Material (Heavy body). These new consistencies are perfect for use with special trays. Putty costs less than £35 and 2x50ml wash less than $£ 21$. A free gun is offered to users buying 2 putty and 2 packs of wash. Reader response number 52

\section{Last call for entries}

Vocational trainees have the chance to win $£ 1000$ in a joint initiative from Dentsply and The Cordent Trust. Final year projects which emphasise the importance of preventative care are now being submitted to Mrs Creek Weir, The Cordent Trust, 68 Knowsley Way, Hildenborough, Tonbridge, Kent, ME15 9LB

The closing date for entries is the 31st July 2003. A judging panel will select a finalist who will receive a cheque for $£ 1000$ and the runner up a cheque for $£ 400$. The presentation of the prizes will take place in London in October. Reader response number 53 